\title{
Experimentally investigation of the CCD binning option in particle imaging velocimetry measurements of laminar flows
}

\author{
B. Akselli ${ }^{1, \star}$ and I. Teke ${ }^{2}$ \\ ${ }^{1}$ Scientific and Technical Research Council of Turkey-National Metrology Institute, TUBITAK-UME, Gebze, Kocaeli, Turkey \\ 2 Department of Mechanical Enginnering, Yildiz Technical University, Istanbul, Turkey
}

Received: 26 February 2011 / Accepted: 12 November 2011

\begin{abstract}
In this work we present an experimental investigation of the employment usage application of the vertical pixel binning (PB) option for PIV (particle image velocimetry) measurements. The PB option increases the speed of a CCD, at the cost of loosing spatial resolution. Consequently, it is expected that PB will positively impact the dynamic velocity range of the PIV measurements. In order to show the benefit of the CCD PB option in PIV measurements, we have carried out series of microPIV experiments on laminar flows, seeded with $1 \mu \mathrm{m}$ fluorescent polystyrene microparticles and passing through a $200 \mu \mathrm{m} \times 200 \mu \mathrm{m} \times$ $50000 \mu \mathrm{m}$ microchannel. The flow images were recorded at normal, $2 \times 1$, and $3 \times 1$ vertical PB modes of a monochrome CCD camera. The experimentally obtained velocity profiles were calculated using the ensemble-averaged cross-correlation method and Gaussian sup-pixel interpolation and then compared with theoretically calculated velocity profiles. We found that the error introduced by the PB option did not exceed the inherent uncertainty of the PIV system used. For a particular PIV system CCD camera, using the PB option allowed an increase in the dynamic velocity range of a PIV system by more than a factor of two, without extra investments.
\end{abstract}

Keywords: PIV (particle image velocimetry); pixel binning; microchannel; flow measurement

\section{Introduction}

PIV is a noninvasive optical measuring technique, which is widely applied for fluid motion analysis [1]. Applications of PIV have extended from measuring the velocity of sand and dusts in tunnels to micro/nanofluid flows in microscale lab-on-a-chip devices [2]. Various modifications of PIV are widely used for flow investigation, detection and control of chemical reactions, sample preparation, characterization of pressure and chemical sensors, microscale systems for biological diagnostics such as DNA analysis by means of PCR (polymerase chain reaction) [3], miniaturized pumping, underwater propulsion systems, and many others [4].

In PIV experiments, the fluid of interest is seeded with fluorescent dyed micrometer or submicrometer scale freefloating probe particles [5-7]. The full field-of-view (FOV) of epifluorescent imaging optics is used to observe a part of the channel illuminated by a sheet of bright light, such as high power pulse lasers with a wavelength corresponding to the fluorescence excitation of the tracer particles. The relative position of particles within the same FOV at a minimum of two instances of time is recorded by employing a high-speed camera. Cross correlation analysis of consecutive frames is performed to measure the average particle displacements within interrogation spots, which

^ Correspondence: basak.akselli@ume.tubitak.gov.tr are then interpolated to ascertain the overall motion, field of the fluid [8-10].

Particle Tracking Velocimetry (PTV) is another widely used approach for flow velocity analysis. The major difference between PTV and PIV is that the later ascertains flow velocity fields by tracking individual particles rather than using cross correlating interrogation volumes, as is done in PIV. The nearest neighbor search with geometrical constraints [11] and binary-image cross correlation between regions around particles of consecutive frames [12] are frequently used particle tracking methods. Initially, PTV was reliably applicable only to sparse seed flows, which was constraining the resolution of the calculated vector fields. With the introduction of 'super-resolution' algorithms, however, [13] flows with much higher particle density can be analyzed by PTV. 'Super-resolution' algorithms perform a two stage flow analysis; first a crosscorrelation is applied to obtain a PIV vector field, which is then used to constrain the search for particle matches in a subsequent PTV step [14]. With the advent of microfluidic and lab-on-a-chip devices the need to measure flows passing through micron-meter scale channels has emerged and brought to existence micron resolution Particle Image Velocimetry (micro-PIV) [15-17], which is a technical modification to PIV. The major difference between PIV and microPIV is in the flow illumination approaches, where the aim is to illuminate the flow in such a way that intensity interference of particles flowing in different velocity layers 
of the flow is reduced, while the image processing techniques and velocity field calculations remain the same. For instance, in a typical PIV experiment a light sheet formed by a laser is used to illuminate only a section of the flow, where the thickness of the light sheet is smaller than the depth of focus of the image recording system. Such illumination approach is impractical for microPIV, as obtaining a stable light sheet of a few micron-meter thicknesses is a technically challenging issue. Instead microPIV applies so-called volume illumination, where the whole volume of the flow is illuminated in, where the FOV and the depth of focus of the microscope objective define the measurement volume.

Initially, PIV setups were designed for experimental research of flow physics and were very expensive and bulky. In most of these setups the illumination was performed by dual cavity lasers, where the output was reaching several mJ at pulse lengths of tens of nanoseconds. Currently, there is a trend towards designing cost-effective and portable PIV systems. For instance, PIV illumination using light emitting diodes (LED) was investigated in [18] and a miniature PIV system with LED illumination is presented in $[19,20]$.

Unfortunately, illumination is not the only parameter that constrains cost and portability of a practical PIV system. For instance, the maximum assessable flow velocity is determined by the sampling frequency (also referred as speed or frame rate) of the imager used to record the flow. One should expect cost of the imager to grow significantly with increasing speed. Double frame cameras, which can capture straddled frames with a nanosecond inter frame delay is an alternative to high speed cameras. Although double frame cameras are much more affordable than the high speed cameras, they require pulse lasers for attaining the desired delays. Due to the high price of pulse lasers, this option is less attractive.

Contemporary CCD cameras allow the use to manipulate their read out mechanisms through the PB option, which increases the frame rate under certain conditions $[21,22]$. This gives a cheap alternative to high speed cameras, but, the speed up comes at the cost of loosing spatial resolution. However, as it was shown in [23], the degradation of the spatial resolution of CCD in binning modes can be compensated by using seeding particles with adequate diameters with respect to the sizes of the super pixel in the binning modes. On the other hand, vertical binning modes preserve CCD resolution in the horizontal direction. Consequently, as it was shown in [23], employing vertical binning modes in PIV measurements in laminar flow (when the horizontal direction of the CCD coincide with the direction of flow) will increase the dynamic velocity range by a factor of at least two, without changing the spatial velocity range in the streamwise direction.

In this work we applied $2 \times 1$ and $3 \times 1$ vertical binning modes in microPIV investigations of the laminar flow inside microchannels with $200 \mu \mathrm{m} \times 200 \mu \mathrm{m}$ cross-section size. By changing the flow rate of the pump, the maximum measurable flow velocities for the normal, $2 \times 1$ and $3 \times 1$ vertical binning modes were defined (Fig. 1).

\section{Dynamic velocity and spatial ranges of PIV}

The dynamic velocity range is the ratio between the maximum and minimum assessable velocity measurements a PIV system can attain under fixed instrumental parameters [24]. This metric defines the upper limit on the resolvable velocity variation within the flow. For instance, the system will be unable to fully ascertain flow field if the dynamic velocity range is less than the magnitude of the velocity variation within the flow. High dynamic velocity range is essential for characterization of turbulent flows, where the velocity drastically varies across the FOV. Contemporary PIV systems achieve dynamic velocity ranges of an order of several hundred, but still this does not suffice for certain types of flows. The dynamic spatial range is defined by the ratio of the FOV in the object space to the smallest resolvable spatial variation [25]. This range corresponds to the number of non-overlapping displacement vector measurements that can be done across the FOV. Higher dynamic spatial range implies better capability to assess small scale variation within the large scale fluid motion. In digital PIV systems the dynamic spatial range is defined by the pixel of a camera, where the smaller pixel size implies better dynamic spatial range. However, covering a certain FOV with small size pixels requires more pixels. The increase of total number of pixels negatively influences the readout time, camera rate, dynamic velocity range of the PIV systems, i.e.

The camera frame rate can be increased, without changing the FOV, by applying one of the PB modes commonly available in modern CCDs. In PB mode the values of adjacent pixels are merged, either horizontally, vertically, or in both directions simultaneously. The merging is done after exposure by special control of the serial and parallel registers in the CCD before the charges are digitized by the on-chip logic [23].

While the FOV is preserved by the PB modes, the spacial resolution is reduced, thus negatively influencing the image quality. It is therefore important to consider the trade-off between frame rate and spacial frequency response when designing optical systems which rely on PB mode. A classical quantitative metric for measuring the performance of an imaging system is the modulation transfer function (MTF). MTF, which is a function of the spatial frequency of an image, quantifies the image contrast $[26,27]$. The influence of the $\mathrm{PB}$ process on the sensor MTF was investigated in [28], where it was shown that with increasing PB orders the total twodimensional MTF of the sensor is decreased. However, as it was shown in $[25,28]$, at vertical PB modes the degradation of the MTF occurred only in the vertical direction while the one-dimensional MTF in the horizontal direction remained unchanged. Since, in laminar flows, the variation of the velocity vector in the direction perpendicular to the streamwise direction is negligible, applying the vertical binning option to PIV measurements of laminar flows will increase the dynamic velocity range, without changing the dynamic spatial range at the stream-wise direction where the velocity gradient is significant. 


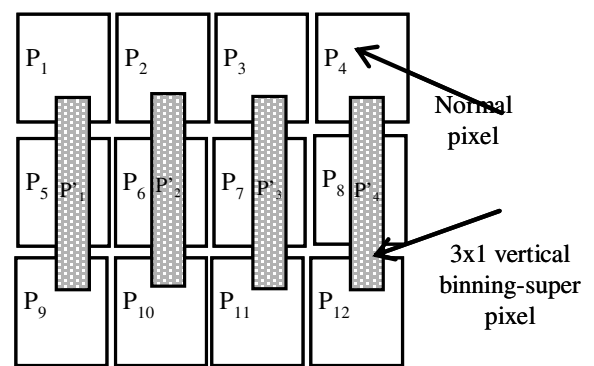

Fig. 1. Schematic representation of the $3 \times 1$ vertical pixel binning.

Table 1. Some parameters of the CCD camera in used PB experiments (FPS-frame per second).

\begin{tabular}{cccc}
\hline Binning & Pixel number & Pixel size $(\mu \mathrm{m})$ & FPS \\
\hline Normal & $1360 \times 1024$ & $4.65 \times 4.65$ & 20.1 \\
$2 \times 1$ & $1360 \times 512$ & $4.65 \times 9.3$ & 33.4 \\
$3 \times 1$ & $1360 \times 341$ & $4.65 \times 13.95$ & 42.2 \\
\hline
\end{tabular}

In the next sections we will describe the experimental setup, which was used in our investigations of the CCD binning option.

\section{Experimental setup}

In this section we describe our experimental setup and flow data acquisition procedure. Figure 2 shows a schematic representation of the experimental setup. A programmable syringe pump (NE-1000, New Era Pump Systems, Inc.) was used to pump the fluid through the microchannel. The flow velocity was regulated by adjusting the output rate of the pump to the desired value. In the experiments, double distilled water was seeded with spherical polystyrene fluorescent particles (Duke Scientific, USA). The density of particles in the liquid is approximately $\rho=1.05 \mathrm{~g} / \mathrm{cm}^{3}$, so they are expected to tag the flow streamlines without excessive slip. Flow measurements were performed using $1 \mu \mathrm{m}$ diameter particles, where the volumetric particle concentration was approximately $0.2 \%$. According to the manufacturer certificate, the standard deviation of the particle size is about $0.011 \mu \mathrm{m}$. The FOV of the microscope was illuminated using a back-lit illumination configuration. The illumination of the high power pulse laser with $532 \mathrm{~nm}$ peak wavelengths was focused on the back side of the glass plate. Imaging was realized by utilizing a Hirox variable zoom lens $(1-10 \times)$ conjugated with a OL-700II objective. In order to record only the fluorescence excitation of the particles and eliminate laser illumination, a long pass filter was placed between the objective and the CCD camera. We investigated steady-state laminar flows in $200 \mu \mathrm{m} \times 200 \mu \mathrm{m} \times 50000 \mu \mathrm{m}$ microchannels. Flows were recorded using a monochrome digital camera, whose PB parameter is given in Table 1 .

The microchannel holder was mounted onto the precise alignment stage in a way that made it possible to adjust the microscope focus onto different depth levels inside the channel. In our experiments, the flow of particles was recorded at a depth of $15 \mu \mathrm{m}$, where the flow in

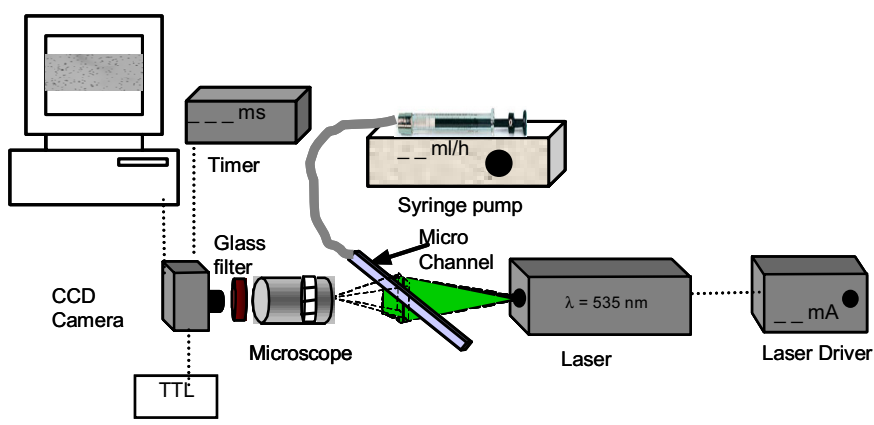

Fig. 2. (Color online) Block-schema of the experimental setup.

question has its maximum gradients. We performed flow recording about $10 \mathrm{~min}$ after starting the pump, so the flow measured inside the microchannel can be considered as a pressure-driven steady-state uncompressed flow. The homemade image recording software was able to record a cycle of PIV images (200 frames) in the normal mode and at the desired binning modes. Just after finishing a cycle the captured frames were stored in uncompressed TIFF format to the hard disc storage, thereby avoiding any delay resulting from computer systems. In order to validate that the flow rate did not change through-out the cycle, the cycle boundary flow profiles were calculated and compared. The boundary profiles correspond to the flow profiles calculated using the full frame PIV image sequences captured at the beginning and the end of the cycle, respectively. Cycles where the difference between the boundary profiles exceeded $2 \%$ were discarded. PIV image analysis was carried out using in-house software written in MATLAB. Measurements were carried out at room temperature, i.e., $23.0 \pm 0.5^{\circ} \mathrm{C}$.

\section{Results and discussions}

\subsection{Sub-pixel peak fitting}

The velocity vector profiles were obtained by ensembleaveraging cross correlation, where, for the best estimate of the particle displacements, a normalized cross correlation function, defined as follows, was used [2]:

$$
\begin{aligned}
& C(u, v)= \\
& \frac{\sum_{x, y}(I(x, y)-\bar{I}(u, v))(T(x-u, y-v)-\bar{T}(u, v))}{\sqrt{\sum_{x, y}(I(x, y)-\bar{I}(u, v))^{2}} \sqrt{\sum_{x, y}(T(x-u, y-v)-\bar{T}(u, v))^{2}}}
\end{aligned}
$$

where, $C(u, v)$ is a normalized cross correlation coefficient of an image $I(x, y)$ (the search area) and a template $T$ (interrogation window in our case), where $T$ is positioned over $I$ at $u, v$ coordinates, $\bar{I}(u, v)$ and $\bar{T}(u, v)$ denote mean values of the images $I$ and $T$, respectively.

The ensemble-averaging cross correlation approach is widely used for displacement field estimation of steady flows. The method is known to greatly increase measurement accuracy in flows containing low particle density, 
flows recorded with insufficient spatial resolution and with a considerable amount of acquisition noise. In the current study, we have employed ensemble averaging of correlation functions, where instantaneous correlation functions of corresponding interrogation regions are averaged prior to the peak location estimations. Correlation averaging has been reported to outperform other proposed averaging methods and less sensitive to erroneous flow measurements [29]. The flow velocity field in each stage was evaluated using ensemble-averaging of 20 instantaneous measurements.

Since the peak in a normalized cross correlation function is located at a discrete location, which is due to the discrete nature of equation (1), the displacement calculated using the normalized cross-correlation function is coarse. In other words, an error of up to \pm 0.5 pixels is present in the correlation peak. In order to increase accuracy of distance estimation in PIV, it is necessary to locate the correlation peak within sub-pixel accuracy. A variety of sub-pixel interpolation methods have been proposed in the literature [30,31]. In [32] a sub-pixel interpolation method, which accounts for non-axially orientated, elliptically shaped particle images or correlation peaks, is proposed.

In this work, the seeding particles have a spherical shape, with a standard deviation which, according to the manufacturer datasheet, does not exceed $0.001 \mu \mathrm{m}$. The best fit to the circular image shapes obtained by the normal binning image acquisition mode is 2D Gaussian interpolation [22]. In order to estimate sub-pixel displacement, a Gaussian surface is fitted to the local neighborhood around the peak of the correlation function. The maximum of the interpolated Gaussian surface is located at $\Delta \mathrm{x}, \Delta \mathrm{y}$ with respect to the index $x_{0}, y_{0}$ of the correlation peak. Assuming the correlation peak has a Gaussiandistribution shape, the accurate location of the correlation peak is:

$$
x=x_{0}+\Delta x \text { and } y=y_{0}+\Delta y
$$

where,

$$
\Delta x=\frac{\ln C\left(x_{0}-1, y_{0}\right)-\ln C\left(x_{0}+1, y_{0}\right)}{\ln C\left(x_{0}-1, y_{0}\right)+\ln C\left(x_{0}+1, y_{0}\right)-2 \ln C\left(x_{0}, y_{0}\right)}
$$

and,

$$
\Delta y=\frac{\ln C\left(x_{0}, y_{0}-1\right)-\ln C\left(x_{0}, y_{0}+1\right)}{\ln C\left(x_{0}, y_{0}-1\right)+\ln C\left(x_{0}, y_{0}+1\right)-2 \ln C\left(x_{0}, y_{0}\right)} .
$$

As noted in [23] any asymmetric binning mode introduces scale distortion to the output image. Any distortion in the image acquisition step, which does not affect the particle location information, can be overcome by determining the nature of this distortion and applying corresponding corrections. For this purpose we have generated a series of air bubbles inside the flow and recorded their images at normal, $2 \times 1$ and $3 \times 1$ binning modes, as shown in Figure 3 . Simple geometrical analysis of the 2D shape of the bubbles indicated that in the binning modes under investigation the circular shape of bubbles degenerated into elliptical shapes.

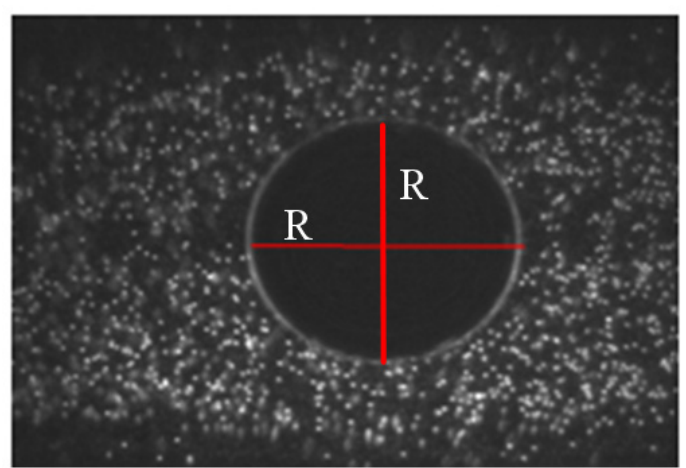

(a)

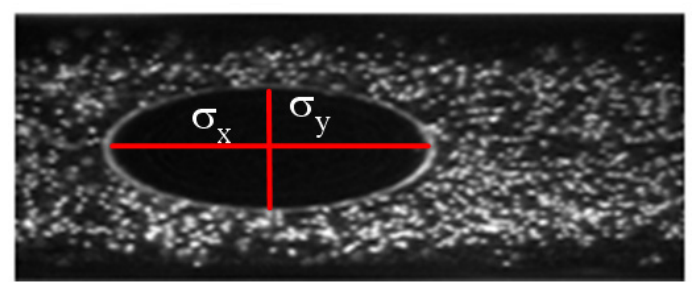

(b)

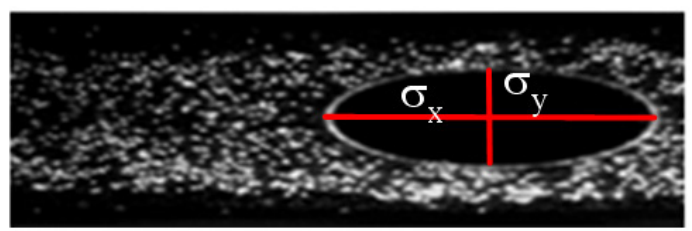

(c)

Fig. 3. (Color online) Bubble images recorded at CCDs: (a) normal mode, (b) $2 \times 1$ vertical binning mode, (c) $3 \times 1$ vertical binning mode.

The general elliptical Gaussian function centered at $(\Delta x, \Delta y)$ is defined as follows [32]:

$$
z(x, y)=A e^{-\left(b_{20}(x-\Delta x)^{2}+b_{11}(x-\Delta x)(y-\Delta y)+b_{02}(y-\Delta y)^{2}\right)}
$$

where $A$ is a constant coefficient,

$$
\begin{aligned}
& b_{11}=\sin ^{2} \theta / 2 \sigma_{x}^{2}+\cos ^{2} \theta / 2 \sigma_{y}^{2} \\
& b_{20}=\cos ^{2} \theta / 2 \sigma_{x}^{2}+\sin ^{2} \theta / 2 \sigma_{y}^{2} \\
& b_{02}=-\sin 2 \theta / 4 \sigma_{x}^{2}+\sin 2 \theta / 4 \sigma_{y}^{2}
\end{aligned}
$$

where $\theta$ is the angle between the $x$ axis (in our case, motion direction) and the major semi-axis, and $\sigma_{x}, \sigma_{y}$ are the major and minor semi-axes of the ellipse, respectively.

We have examined the dimensions of the bubbles in Figure 3. and found that: (a) in the normal capturing mode the shape of a single particle is a circle with radius $\sigma_{x}=\sigma_{y}=R$, (b) in $2 \times 1$ binning mode the shape is transformed into horizontal ellipse with $\theta=0$ and $\sigma_{x} / \sigma_{y}=2$, (c) in $3 \times 1$ binning mode $\theta=0$ and $\sigma_{x} / \sigma_{y}=3$.

In [28] a general elliptical Gaussian function for the sub-pixel interpolation for the cases of elliptically shaped, non-axially oriented particle images or correlation peaks is proposed, where the coordinates of the maximum peak location were derived using a compact form of elliptical 


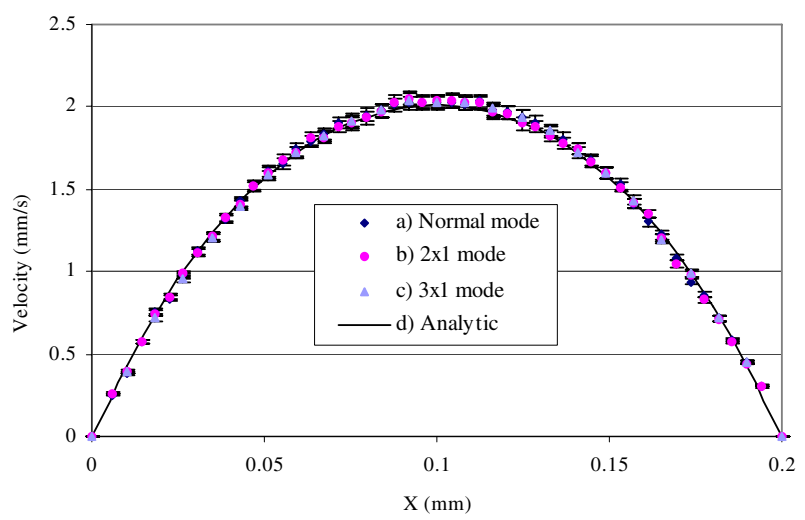

Fig. 4. (Color online) Velocity profiles (a) normal mode, (b) $2 \times 1$ vertical binning mode; (c) $3 \times 1$ vertical binning mode; (d) Solid curve depicts the analytically calculated velocity profile.

Gaussian intensity function (Eq. (7)) and two-dimensional regression analysis:

$$
z(x, y)=e^{-\left(c_{00}+c_{10} x+c_{20} x^{2}+c_{01} y+c_{11} x y+c_{02} y^{2}\right)} .
$$

In the cases of vertical and horizontal binning modes, the particle images obtained and their corresponding correlation peaks are deformed into elliptical shapes, but are still axially oriented (along the $y$ and $x$ axes, respectively). Considering these factors (7) can be constrained to the axially oriented particle images or correlation peaks by setting coefficient $c_{11}$ to 0 :

$$
z(x, y)=e^{-\left(c_{00}+c_{10} x+c_{20} x^{2}+c_{01} y+c_{02} y^{2}\right)} .
$$

At the vertical $2 \times 1$ binning mode the particle images and corresponding correlation peaks are oriented along the $y$ axis and are deformed (by a 1:2 ratio). By applying twodimensional regression, it can be shown that the sub-pixel location of the peak is reformulated as:

$$
x=x_{0}+\Delta x \text { and } y=y+\Delta y / 2
$$

where $\Delta x$ and $\Delta y$ are defined as in equations (3) and (4), respectively.

In a similar way, for the vertical, $3 \times 1$ binning mode, where particle images and corresponding correlation peaks are oriented along the $y$ axis and are deformed (by a 1:3 ratio), the sub-pixel location of the peak is reformulated as:

$$
x=x_{0}+\Delta x \text { and } y=y+\Delta y / 3 .
$$

\subsection{Measurement results}

Figure 4 shows the comparison of the three experimental velocity profiles obtained in the square michrochannel at three different PB modes of the CCD: (a) normal mode, (b) $2 \times 1$ Binning mode, (c) $3 \times 1$ binning mode. The maximum velocity is about $2.05 \mathrm{~mm} / \mathrm{s}$. By changing the pump output value and analysing the results of cross-correlation

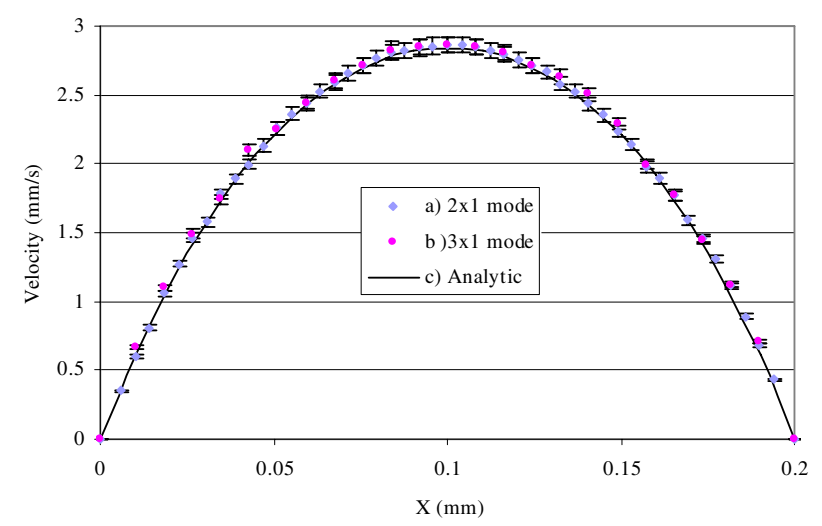

Fig. 5. (Color online) Velocity profiles (a) $2 \times 1$ vertical binning mode, (b) $3 \times 1$ vertical binning mode, (c) Solid curve depicts the analytically calculated velocity profile.

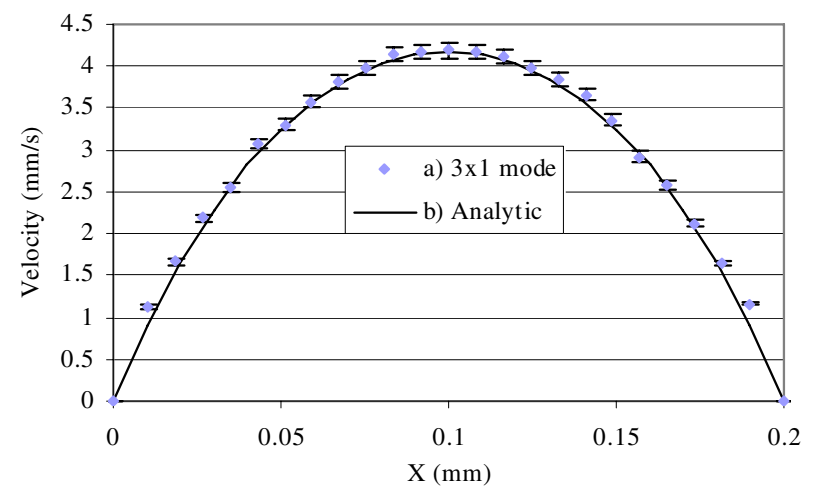

Fig. 6. (Color online) Velocity profiles, (a) $3 \times 1$ vertical binning mode, (b) solid curve depicts the analytically calculated velocity profile.

calculations, we established that the velocity value is the maximum velocity value, which can be measured at the normal mode of our CCD camera and particular microPIV system.

The analytical velocity profile in Figures 4-6 (solid curve) was obtained using the following equations [33]:

$$
\begin{aligned}
u_{1}(y, z)= & \frac{16 a^{2}}{\mu \pi^{3}}\left(-\frac{d p}{d x}\right) \sum_{i=1,3,5, \ldots}^{\infty}(-1)^{(i-1) / 2} \\
& \times\left[1-\frac{\cosh (i \pi z / 2 a)}{\cosh (i \pi b / 2 a)}\right] \frac{\cos (i \pi y / 2 a)}{i^{3}} \\
Q= & \frac{4 b a^{3}}{3 \mu}\left(-\frac{d p}{d x}\right) \\
& \times\left[1-\frac{192 a}{\pi^{5} b} \sum_{i=1,3,5, \ldots}^{\infty} \frac{\tanh (i \pi b / 2 a)}{i^{5}}\right]
\end{aligned}
$$

where $Q$ is the flow rate, $a$ and $b$ are the dimensions of the cross-section of the cannel, and $\mu$ is fluid viscosity.

In general, the measurement uncertainties of PIV and micro-PIV are the same, except for those arising from the difference in illumination techniques, namely sheet and volume illuminations. The major source of error in microPIV is the definition of the depth of field. In the micro-PIV 
literature there are discrepancies between definitions for the depth of field of an imaging microspore. In our calculations, we have used the equation described in [34]:

$$
\delta z_{m}=\frac{3 n \lambda}{N A^{2}}+\frac{2.16 d_{p}}{\tan \theta}+d_{p}
$$

where $\mathrm{n}$ is the refractive index of the medium, and $N A$ is the numerical aperture of the objective lens. The depth of field calculated according to (13) is equal to $2.7 \mu \mathrm{m}$. This value indicates that the intensity contributed by particles out of this focus depth has a negligible influence on the correlation function. The size of the interrogation windows were $60 \times 30,60 \times 15$ and $60 \times 10$ pixels for normal, $2 \times 1$, and $3 \times 1$ vertical binning modes, respectively. Consequently, interrogation volumes in the flow coordinates were the same for all binning modes and equal to $23.25 \mu \mathrm{m} \times 5.8 \mu \mathrm{m} \times 2.7 \mu \mathrm{m}$. The interrogation spots were overlapped by $50 \%$ to satisfy the Nyquist sampling criterion, which corresponds to $2.4 \mu \mathrm{m}$ vector-to-vector spacing in the stream-wise-normal direction.

In the normal mode, a $1 \mu \mathrm{m}$ particle was resolved by approximately 4 pixels, which is in accord with the minimum requirement (3-4 pixels) essential to obtain the location of a particle image correlation peak to within one tenth of the particle's image diameter [14]. Accordingly, the measurement uncertainty can be determined using $d_{x} \approx d_{e} / 10 M$, which yields $0.14 \mu \mathrm{m}$ for our experiments. For a velocity of $0.7 \mathrm{~mm} / \mathrm{s}$ and a time between two frames of $0.05 \mathrm{~s}$, the error was evaluated to be approximately $0.7 \%$.

The error due to particle diffusion caused by Brownian motion along the $x$-axis is given by [35]:

$$
\varepsilon_{B}=\sqrt{2 D /\left(u^{2} \Delta t\right)}
$$

where $D$ is the Brownian diffusion coefficient:

$$
D=\frac{k T}{3 \pi \mu d_{p}} .
$$

In equation (15), $k=1.38 \times 10^{-23} \mathrm{~J} / \mathrm{K}$ is Boltzman's constant, $T$ denotes fluid temperature, $d_{p}$ is the particle diameter, and $\mu$ is the dynamic viscosity of the fluid. For the current experiments with characteristic flow velocity of $u \approx 0.7 \mathrm{~mm} / \mathrm{s}$ and $\Delta t=0.05 \mathrm{~s}$, this approximately yields a $0.5 \%$ relative error due to Brownian motion.

The settling velocity $u$ of the particle under gravity, assuming that the process is governed by Stokes drag, is given by (for example, [5]):

$$
u=\frac{g d_{p}^{2}\left(\rho_{p}-\rho_{f}\right)}{18 \mu}
$$

where, $d_{p}$ is particle diameter, $\rho_{p}$ and $\rho_{f}$ are particle and fluid density, respectively, and $\mu$ is fluid viscosity. The requirement is for $u$ to be as small as possible in comparison to the actual flow velocity. As can be seen from equation (16) particles from materials with densities close to the fluid density are suitable for flow visualization. In our experiments with polystyrene particles with a density of $\rho_{p}=1.05 \mathrm{~g} / \mathrm{cm}^{3}$ equation (16) leads to a value of $u \approx 2.7 \times 10^{-5} \mathrm{~mm} / \mathrm{s}$, which is sufficiently smaller then the measured fluid velocities.

The stability of the system, including the syringe drive pump, was estimated using the standard deviation of mean velocities calculated using the central part of the velocity profiles of different PIV measurement cycles. The stability was found to be within $2 \%$ of the maximum mean velocity of the normal mode.

By further increasing the velocity of pump output step by step we established the maximum velocity of flow which can be measured at $2 \times 1$ binning mode. For the particular CCD and micro-PIV system we found this value to be $2.86 \mathrm{~mm} / \mathrm{s}$. Figure 5 depicts the experimentally obtained laminar flow velocity profiles at a maximum velocity value of $2.86 \mathrm{~mm} / \mathrm{s}$ at $2 \times 1$ and $3 \times 1$ binning modes. The ratio of maximum assessable velocities at the $2 \times 1$ binning mode and the normal mode is about 1.5, which shows that the dynamic velocity range of the micro-PIV system at $2 \times 1$ mode is increased (by a factor of 1.5).

Finally, Figure 6 shows the velocity profile measured at $3 \times 1$ vertical binning mode. The maximum assessable velocity value is $4.18 \mathrm{~mm} / \mathrm{s}$. The ratio of maximum velocities at the $3 \times 1$ binning mode and normal mode is about 2 , which is in good agreement with the increased frame rate of the camera at $3 \times 1$ binning mode relative to normal mode (Tab. 1).

\section{Conclusion}

The CCD PB option offers new possibilities in particle image velocimetry for increasing the dynamic velocity range of measurements. In this work we experimentally studied the benefits of using the PB option in PIV measurements. We experimentally defined the maximum value of the measurable velocity of laminar flow inside a microchannel. First we compared the velocity profiles obtained at tree different binning modes (at the normal mode, at the $2 \times 1$ and $3 \times 1$ vertical binning modes) at slow flow velocities (maximum value about $2 \mathrm{~mm} / \mathrm{s}$ ), and showed that the discrepancy between velocity profiles does not exceed the inherent error of the utilized micro PIV system. Then, by gradually increasing the flow rate while monitoring the observed velocity profiles, we determined the maximum measurable velocities to be $4.5 \mathrm{~mm} / \mathrm{s}$ and $3 \mathrm{~mm} / \mathrm{s}$ for $3 \times 1$ and $2 \times 1 \mathrm{~PB}$ modes, respectively, compared to $2 \mathrm{~mm} / \mathrm{s}$ in normal mode with the same camera.

\section{References}

1. R.J. Adrian, Particle-imaging techniques for experimental fluid mechanics, Annu. Rev. Fluid Mech. 23, 261-304 (1991)

2. M. Raffel, C.E. Willert, S.T. Wereley, J. Kompenhans, Particle Image Velocimetry - A Practical Guide, 2nd edn. (Springer, 2007) 
3. D. Erickson, D. Li, Integrated microfluidic devices, Anal. Chim. Acta 507, 11-26 (2004)

4. S.D. Peterson, M. Porfiri, A. Rovardi, A particle image velocimetry study of vibrating ionic polymer metal composites in aqueous environments, IEEE/ASME Trans. Mechatron. 14, 474-483 (2009)

5. H. Nasibov, S. Baytaroglu, Recent advances in digital particle image velocimetry methods for flow motion analysis, Int. J. Metrol. Qual. Eng. 1, 21-29 (2010)

6. R.J. Adrian, Twenty years of particle image velocimetry, Exp. Fluids 39, 159-169 (2005)

7. M. Honkanen, H. Nobach, Background extraction from double-frame PIV images, Exp. Fluids 38, 348-362 (2005)

8. C.E. Willert, M. Gharib, Digital particle image velocimetry, Exp. Fluids 10, 181-193 (1991)

9. A.K. Prasad, R.J. Adrian, C.C. Landreth, P.W. Offutt, Effect of resolution on the speed and accuracy of particle image velocimetry interrogation, Exp. Fluids 13, 105-116 (1992)

10. F. Scarano, Iterative image deformation methods in PIV, Meas. Sci. Technol. 13, R1-R19 (2002)

11. Y. Hassan, R. Canaan, Full-field bubbly flow velocity measurements using a multiframe particle tracking technique, Exp. Fluids 12, 49-60 (1991)

12. P. Ruhnau, C. Guetter, T. Putze, C. Schnorr, A variational approach for particle tracking velocimetry, Meas. Sci. Technol. 16, 1449-1458 (2005)

13. R.D. Keane, R.J. Adrian, Y. Zhang, Super resolution particle image velocimetry, Meas. Sci. Technol. 6, 754-768 (1995)

14. M.R. Bown, J.M. MacInnes, R.W.K. Allen, W.B.J. Zimmerman, Three-dimensional, three-component velocity measurements using stereoscopic micro-PIV and PTV, Meas. Sci. Technol. 17, 2175-2185 (2006)

15. J.G. Santiago, S.T. Wereley, C.D. Meinhart, D.J. Beebe, R.J. Adrian, A particle image velocimetry system for microfluidics, Exp. Fluids 25, 316-319 (1998)

16. C.D. Meinhart, S.T. Wereley, J.G. Santiago, PIV measurements of a microchannel flow, Exp. Fluids 27, 414-419 (1999)

17. S. Devasenathipathy, J.G. Santiago, S.T. Wereley, C.D. Meinhart, K. Takehara, Particle imaging techniques for microfabricated fluidic systems, Exp. Fluids 34, 504-514 (2003)

18. H. Nasibov, A. Kholmatov, B. Akselli, A. Nasibov, Experimental study of digital micro-particle-imagevelocimetry $(\mu \mathrm{PIV})$ system with $\mathrm{LED}$ illumination, International Conference of Metrology (CAFMET 2010) (Cairo, Egypt, 2010)

19. O. Chételat, K.C. Kim, Miniature particle image velocimetry system with LED in-line illumination, Meas. Sci. Technol. 13, 1006-1013 (2002)
20. S.M. Hagsäter, C.H. Westergaard, H. Bruus, J.P. Kutter, Investigations on LED illumination for micro-PIV including a novel front-lit configuration, Exp. Fluids 44, 211-219 (2008)

21. J.R. Janesick, Scientific Charge-Coupled Devices (SPIE Optical Engineering Press, Washington, USA, 2001)

22. B. Akselli, A. Kholmatov, H. Nasibov, The use of CCD pixel binning in PIV measurements, International Symposium on Optomechantronic Technologies (ISOT 2009) (Istanbul, Turkey, 2009), pp. 223-228

23. H. Nasibov, A. Kholmatov, B. Akselli, A. Nasibov, S. Baytaroglu, Performance analysis of the CCD pixel binning option in particle image velocimetry measurements, IEEE/ASME Trans. Mechatron. 15, 527-540 (2010)

24. R.J. Adrian, Dynamic ranges of velocity and spatial resolution of particle image velocimetry, Meas. Sci. Technol. 8, 1393-1398 (1997)

25. A. Kholmatov, B. Akselli, A. Nasibov, H. Nasibov, Subpixel centroid position error analysis in particle tracking velocimetry induced by the CCD pixel binning, Proc. SPIE 7723, 77231R (2010)

26. G.D. Boreman, Modulation Transfer Function in Optical and Electro-Optical Systems (SPIE Press Ltd, US, 2001)

27. A. Nasibov, A. Kholmatov, H. Nasibov, F. Hacizade, Investigation of a CCD modulation transfer functionsing the speckle method at different laser wavelengths and subwindowing options, Int. J. Metrol. Qual. Eng. 2, 25-30 (2011)

28. A. Nasibov, A. Kholmatov, H. Nasibov, F. Hacizade, The influence of CCD pixel binning option to its modulation transfer function, Proc. SPIE 7723, 77231a (2010)

29. C.D. Meinhart, S.T. Wereley, J.G. Santiago, A PIV algorithm for estimating time-averaged velocity fields, ASME Trans. J. Fluids Eng. 122, 285-289 (2000)

30. M.R. Cholemari, Modeling and correction of peak-locking in digital PIV, Exp. Fluids 42, 913-922 (2007)

31. H. Nobach, N. Damaschke, C. Tropea, High-precision subpixel interpolation in particle image velocimetry image processing, Exp. Fluids 39, 299-304 (2005)

32. H. Nobach, M. Honkanen, Two-dimensional Gaussian regression for sub-pixel displacement estimation in particle image velocimetry or particle position estimation in particle tracking velocimetry, Exp. Fluids 38, 511-515 (2005)

33. F.M. White, Viscous Fluid Flow (McGraw Hill, New York, 2006)

34. C.D. Meinhart, S.T. Wereley, M.H.B. Gray, Volume illumination for two-dimensional particle image velocimetry, Meas. Sci. Technol. 11, 809-814 (2000)

35. M.G. Olsen, C.J. Bourdon, Out-of-plane motion effects in microscopic particle image velocimetry, ASME Trans. J. Fluids Eng. 125, 895-901 (2003) 